XVII.

Aus der I. inneren Abtheilung des Stadtkrankenhauses Friedrichstadt zu Dresden. Oberarzt Prof. Dr. Ad. Schmidt.

\title{
Ein Beitrag zur pathologischen Anatomie der Landry'schen Paralyse.
}

\author{
Von
}

Dr. Hans Kohrisch,

Die von Landry') im Jahre 1859 zuerst beschriebene, von ihm „paralysie ascendante aigue" genannte Erkrankung beansprucht auch beute noch in unvermindertem Maasse das Interesse der Kliniker und Pathologen, zumal die Ansichten' über den Verlauf und besonders über das anatomische Substrat der Krankheit noch sehr getheilte sind. Der Landry'sche Fall war bekanntlich ausgezeichnet durch eine schnell verlaufende aufsteigende Lähmung, wobei Muskelatrophien fehlten und die elektrische Erregbarkeit normal war; es bestanden dabei nur leichte Sensibilitätsstörungen. Der mit den damaligen Hilfsmitteln erhobene mikroskopisehe Befund war negativ. Das Vorkommen dieser Erkrankung mit dem beschriebenen Verlauf wurde späterhin besonders von Westphal ${ }^{2}$ ) bestätigt. Dieser beseichnete auf Grund von 4 genau untersuchten Fällen als charakteristische Symptome der acuten aufsteigenden Spinallähmung: den progressiven aufsteigenden schliesslich tödtlichen Verlauf der Lähmung, das Intaktbleiben der elektrischen Erregbarkeit in den gelähmten Muskeln und den völlig negativen Autopsiebefund. Die bis dahin bekannt gewordenen Fälle mit positiven anatomischen Befunden wurden von Westphal nicht als beweisend anerkannt. Als ätiologisches Moment glaubte Westphal eine Intoxication annehmen zu

1) Note sur la paralysie ascendante aigue. Gaz. hebd. 1859.

2) Ueber einige Fälle von acuter tödtlicher Spinallähmung. Arch. f. Psych. u. Nervenkrankheiten. VI. Band. 1876. S. 765. 
müssen. So schien das Krankheitsbild der Landry'schen Paralyse in klinischer und anatomischer Hinsicht einen gewissen Abschlinss gefunden zu haben, bis sehr bald neue Beobachtungen bekannt wurden, welche geeignet waren, die bestehenden Anschauungen über klinischen Verlauf und pathologische Anatomie dieser eigenartigen Krankheit umzustossen. Ohne auf die einzelnen Fälle näher eingehen zu wollen (cf. Casuistik bei Leyden1), will ich nur erwähnen, dass man klinisch auch absteigenden Verlauf der Lähmung beobachtete, ferner schwere Störungen der elektrischen Muskelerregbarkeit, Blasen- und Mastdarmstörungen, Fieber. In anatomischer Hinsicht wurden zwei Anschauungen verfochten: Die einen glaubten auf Grund nachgewiesener myelitischer Veränderungen in Rückenmark und Medulla oblongata eine Affection des Centralnervensystems annehmen zu sollen (Eisenlohr'2), Leyden ${ }^{3}$ ), v. d. Velden ${ }^{4}$ u. A.), nachdem bereits früher Petit fils ${ }^{5}$ ) die Erkrankung als Poliomyelitis ant. acutissima bezeichnet hatte. Eine andere Gruppe von Autoren neigten dazu, die Landry'sche Paralyse als eine Affection der peripheren Nerven im Sinne einer Polyneuritis. aufzufassen, besonders nach Eichhorst's ${ }^{6}$ ) bekannter Publication. Zunächst freilich stiessen die Beobachtungen mit abweichendem klinischen Verlauf und positiven anatomischen Befunden vielfach auf Widerspruch. So glaubte, um nur einige Autoren zu citiren, Erb7) schon aus klinischen Gründen diese Fälle von der Landry'schen Paralyse trennen zu sollen; er betonte dies mit Entschiedenheit für den Eich horst'schen Fall. Seiner Ansicht nach ist die Landry'sche Paralyse „eine klinisch wohl charakterisirte - allerdings wohl unzweifelhaft spinale - Krankheitsform", bei der es sich um impalpable Ernährungsstörungen im Rückenmark handele. Remaks) hält im Allgemeinen an dem von Landry und Westphal gegebenen Verlauf fest und betrachtet die. Fälle mit gestörter elektrischer Erregbarkeit für spino-peripherische Lähmungen

1) Ueber multiple Neuritis und acute aufsteigende Paralyse nach Influenza. Zeitschr. f. klin. Medicin. 24. Bd. 1894. S. 1.

2) Ein Fall von Paralysis ascendens acuta. Virchow's Archiv. 73. Bd. 1878. S. 73 .

3) 1. c.

4) Ein Fall von acuter aufsteigender spinaler Paralyse. Deutsch. Arch.

f. klin. Med. Bd. 19, Heft 4. 1877.

5) Considérations sur l'atrophie aigue des cellules motrices. Paris 1873.

6) Virch. Archiv. 1876. Bd. 69.

7) Krankheiten des Rückenmarks. Ziemssen, Spec. Pathol. und Therapie. 1876.

8) Eulenburg's Realencyclopaedie. Bd. 18. 1889. 
und als solche nicht zur Landry'schen Paralyse gehörig. Abgesehen von den Fällen mit negativem Befund würde er am ehesten noch eine primäre acute Erkrankung der Medulla oblongata gelten lassen. Aetiologisch findet er vorläufig keine andere Erklärung als die einer noch räthselhaften Intoxication des Nervensystems. Auf Grund eigener und fremder Untersuchungen erweiterte Strümpell ${ }^{\sharp}$ ) das klinische Bild der Erkrankung: er beobachtete Fieber, rasche Abnahme der elektrischen Erregbarkeit, geringe Blasenstörungen, Sensibilitätsstörungen. Er glaubt an eine acute Infection des Körpers mit vorherrschender Localisation im motorischen Nervensystem und hält es für wahrscheinlich, wenn auch nicht für bewiesen, dass die Landry'sche Paralyse die acuteste Form einer multiplen Neuritis ist. v. Leyden und Goldscheider ${ }^{2}$ ) unterscheiden auf Grund der mannigfachen anatomischen Befunde eine bulbäre oder medulläre und eine neuritische Form. Beide Formen können auch combinirt sein, insofern als der Process von den peripheren Nerven zum Rückenmark aufsteigen kann. Nach Soltmann's ${ }^{3}$ ) Ansicht ist eine Trennung der Fäle von Landry'scher Paralyse in solche, die zur Polyneuritis, solche, die zur Myelitis gehören und solche mit negativem Befund unwesentlich und unnöthig. Diese Fälle stellen vielmehr nur verschiedene Stadien ein und desselben Processes dar, nümlich einer Intoxication resp. Infection, je nach Intensität und Dauer derselben. In ganz ähnlicher Weise spricht sich schliesslich auch Oppenheim(4) aus. Er hält eine Scheidung in bulbäre, spinale und periphere Formen für undurchführbar, glaubt vielmehr, dass eine Infection resp. Intoxication erfolgt, welche das motorische Nervensystem befallt und bald in der Peripherie, bald im Centrum, bald in beiden zusammen localisirt ist und zuweilen mikrokospisch sichtbare Spuren hinterlässt.

Im Gegensatz zu diesen Autoren steht eine Publication von Rolly ${ }^{5}$ ) aus dem Jabre 1903. Rolly schildert 7 Falle, welche er nach ihrem klinischen Verlauf als Landry'sche Paralyse bezeichnet, von denen 3 zur Section kamen. Im Fall 1 fand keine genaue mikro-

1) Die sogenannte acute aufsteigende Spinalparalyse. Spec. Pathol. und Ther. Bd. 3. 1897 .

2) Landry'sche Lähmung. Nothnagel's spec. Pathol. und Ther. X. Bd. II. Theil. 1897. S. 440 .

3) Ueber Landry'sche Paralyse. Jahrbuch f. Kinderheilk. Bd. 51. S.67.

4) Lehrbuch der Nervenkrankheiten. Berlin 1902.

5) Zur Kenntniss der L andry' schen Paralyse. Münchener med. Wochenschrift. 1903 . No. 30 und 31 . 
skopische Untersuchung statt, im Fall 2 wurden mikroskopisch keine krankhaften Veränderungen nachgewiesen, im Fall 7 wurde das Centralnervensystem normal befunden, aber neuritische Veränderungen in den feinsten Muskelästen der peripheren Nerven entdeckt. Rolly trägt auf Grund dieses letzten Befundes kein Bedenken, seine sämmtlichen Fälle Landry'scher Paralyse als \Polyneuritis aufzufassen. Er hält es für wahrscheinlich, „dass die schädliche Noxe zuerst nur auf die feinsten Nerven, dic zugleich am empfindlichsten $z u$ sein scheinen, bei den meisten Fällen von Landry'scher Paralyse einwirkt". Im Anschluss hieran entwickele sich eine Neuritis, die offenbar centripetal sich ausbreiten und in manchen Fällen das Rückenmark angreifen könne. „Auf diese Weise liessen sich auch alle anderen Fälle von sogenannter myelitischer Form der Landry'schen Paralyse der Litteratur erklären." Dje Landry'sche Paralyse sei deshalb nicht mehr als eine Krankheit sui generis zu betrachten, sondern als eine Unterart der Polyneuritis acuta. Hierzu muss bemerkt werdea, dass im Fall 7 der Befund neuritischer Veränderungen nach dem klinischen Bild durchaus erklärlich ist und wohl auch in den Fällen 3-6 erhoben worden wäre. Fraglich aber erscheint es, ob sämmtliche 7 Fälle nach ihrem klinischen Verlaufe ïberhaupt der Landry'schen Paralyse zuzuzählen sind. Wem man die Berechtigung dazu für die Fälle 1 und 2 obne Weiteres zugeben muss, so scheint sie für die übrigen Fälle, besonders für 3,4 und 7 nicht unbedingt gegeben zu sein. Rolly selbst hat seine Krankengeschichten mit denen von Polyneuritiden verglichen und gefunden, "dass in der That sehr viele Aehnlichkeiten dieser beiden Krankheiten bestehen", "dass es klinisch mancbmal sehr schwer fallen dürfte, sich für Neuritis acuta oder Landry'sche Paralyse zu entscheiden". Tnd so ist eigentlich nicht einzusehen, warum man die genannten Fälle nicht auch klinisch von vornherein als Polyneuritis acuta auffassen sollte, zumal diese Erkrankung die bei weitem hänfigere ist. Sodann würde auch der verhältnissmässig langsame Verlauf, die schweren Störungen der elektrischen Erregbarkeit, das Auftreten von Muskelatrophien, die Druckempfindlichkeit der Nervenstämme vielmehr für Polyneuritis sprechen als für Landry'sche Paralyse, für die noch immer der schnelle stürmische Verlauf, wie z. B. in dem schönen Soltmann'schen ${ }^{1}$ ) Fall, charakteristisch ist. Es erscheinen deshalb die bereits erwähnten Schlussfolgerungen Rolly's über das Verhältniss der Landry'schen Paralyse zur Polyneuritis, wenigstens in der von ihm angenommenen Allgemeinheit, doch wohl als zu weitgehend, besonders

1) l. c. 
auch im Hinblick auf die zahlreichen Beobachtungen von positiven anatomischen Befunden im Centralnervensystem. Ganz besonders interessant ist in dieser Beziehung eine Beobachtung ron Arneth') ans jüngster Zeit. Die Sectionsdiagnose dieses Falles (33jühriger Mann, der binnen 3 Tagen an Landry'scher Paralyse zu Grunde ging) lautet in Bezug auf das Centralnervensystem: Malacia medullae spinalis dorsalis inferioris circumseripta cum hyperaemia diffusa medullae et cerebri. Arneth bält die Landry'sche Paralyse für eine Infectionskrankheit und verweist dabei auf die grosse Aehnlichkeit mit dem Tetanus, eine Analogie, auf die übrigens bereits von $\mathrm{Erb}^{2}$ ) aufmerksam gemacht wird. Arnetb konnte nun auf Grund des übereinstimmenden Verhaltens der neutrophilen Leukocyten in der Agone bei Landry'scher Paralyse und Tetauus gewichtige Anhaltspunkte dafür finden, dass hier wie dort das Centralnervensystem den Angriffspunkt des infectiösen Virus bildet. Er meint, „wir werden kaum fehlgehen, wenn wir auch dem Nerven lähmenden Gifte der Landry'schen Paralyse eine ausschliessliche Affinität zu den Bestandtheilen der nervösen Centralorgane vindiciren". Seine anatomischen Befunde in Gehirn und Rückenmark, die noch ausführlich publicirt werden sollen, scheinen diese Ansicht zu bestätigen.

Angesichts dieser vielfachen Gegensätze in den Anschauungen über Klinik und pathologische Anatomie der Landry'schen Paralyse muss jeder neue Fall, dessen anatomische Untersuchung möglich ist, Interesse erwecken. Ich möchte daher in Folgenden einen Fall beschreiben, der auf der I. inneren Abtheilung des Friedrichstädter Krankenhauses in Dresden zur Beobachtung kam, und der noch dadurch ausgezeichnet war, dass die Erkrankung eine Person betraf, die bereits an Tabes dorsalis litt.

Die 48jährige Patientin wurde am 21. Juli 1903 in's Krankenbaus aufgenommen. Nach ihren Angaben hatte sie als Kind Scharlach und Nervenfieber gehabt. Sie war von Beruf Kellnerin. Ihr Mann, mit dem sie 10 Jahre verheirathet war, war Kellner. Der Ehe entstammten keine Kinder. Fehlge. burten kamen nicht vor. Lues wird in Abrede gestelit.

Das Leiden, wegen dessen Pat. das Krankenhaus aufsucht, besteht seit 4 Jahren. Es begann mit Gefühl von Schwere und Schwäche in den Beinen, Kribbeln und pelzigen Gefühl in den Füssen. Diese Beschwerden wurden im Laufe der Jahre immer stärker. Es stellten sich schiessende Schmerzen in den Beinen und Druckgefühl um die Brust ein. Vor 6 Wochen traten zum 1. Mal

1) Die agonale Leukocytose. Münchener med. Wochenschr. 1@04. No.27.

2) $1 . c$. 
anfallsweise starke Leib- und Magenschmerzen verbunden mit häufigem Erbrechen ein. Diese Anfälle haben sich seitdem oinige Male wiederholt.

Es handelt sich um eine mittelgrosse, mässig genährte blasse, etwas stupide Person. Die Temperatur ist normal. Herz, Lungen and Nieren sind intact. Leber und Milz sind nicht zu fühlen. Der ganze Leib, besonders die Magengegend, ist etwas druckempfindlich. Eine Dilatation des Magens ist nicht nachzuweisen.

Beide Pupillen sind stecknadelliopfgross ohne jede Reaction. Pat. giebt an, sie könne seit einiger Zeit schleeht sehen. Sie kann Finger und Licht auf weite Entfernungen unterscheiden. Leseproben sind nicht anzustellen, da Pat. nicht lesen kann. Die Hirnnerven sind sämmtlich intact: Gehör, Geschmack, Sprache, Schlucken durchaus normal. Die Zunge wird gerade herausgestreckt. Der Gaumen hebt sich gut. Die rohe Kraft der Arme ist bei gat entwickelter Muskulatur sehr gut erhalten. Die Kraft der Beine ist herabgesetzt. Pat. kann die ausgestreckten Beine nur um ca. $4 j^{0}$ von der Unterlage erheben. Gehen und Stehen ist mit Unterstützung möglich. Der Gang ist schwankend; die Beine werden dabei steif gehalten und zeigen deutliche atactische Bewegungen. Starker Romberg.

In den Füssen und Fingerspitzen bestehen Parästhesien leichten Grades. Objectivsensibilitätsstörungen sind nur an den Beinen nachzuweisen: Das Gefühl für leichte Berührung ist gut erhalten; dagegen ist an beiden Beinen, besonders an den Unterschenkeln, die Algesie stark abgeschwächt. Stellenweise ist die Schmerzemplindung stark verlangsamt. Wärme und KäIte werden richtig empfunden.

Fusssohlen- and Bauchdeckenreflexe sind vorhanden. Patellar- und Achillessehnenreflexe fehlen. Blase und Mastdarm sind intact.

Die Muskulatur des Rumpfes ist kräftig. Pat. kann sich leicht aufsetzen.

Die Nacken- und Halsdrüsen sind zam Theil vergrössert und indurirt.

Es wurde auf Grund dieser Befunde die Diagnose "Tabes dorsalis" gestellt. Während der nächsten Tage blieb der Zustand unverändert. Anfallsweise traten gastrische Krisen auf, mit häufigem Erbrechen einhergehend. Das Erbrochene war meist sebr reichlicb, von bräunlicher Farbe mit Geruch nach Buttersänre. Gesammtacidität 4S. Keine freie $\mathrm{HCl}$. Milchsäure stark positiv. Mikroskopisch Milchsäurebacillen.

Augenhintergrund normal.

26. Juli. Seit heute wird eine in auffallender Weise zunehmende Schwäche der Beine bemerkt bei sonst unverändertem Befund.

28. Juli. Die rohe Kraft der Beine ist jetzt gleich Null; es können nur noch die Zehen etwas bewegt werden. Heben der Beine ist unmöglich. Pat. ist seit gestern sehr weinerlich. Sie kann sich nicht mehr aufsetzen. Gleichzeitig macht sich auch eine deutliche Abnahme der rohen Kraft der Arme bemerkbar. Auch scheint es, als ob Schluckbeschwerden vorhanden wären, wenigstens rerschluckt sich Pat. beim Trinken von Flüssigkeiten sehr leicht. Der Gaumen hebt sich nur wenig.

29. Juli. Beine völlig gelähmt. Aufsetzen unmöglich. Starlie Schwäche 
der Arme. Deatliche Schlucklähmung. Sprache leise, langsam, undeutlich. Kein Eieber.

31. Jali. Die Arme werden kaum noch bewegt. Pat. ist jetzt etwas unklar. Sprechen macht ihr sichtlich Mühe. Die Sprache ist leise, schwerfällig, aussetzend. Ausgesprochene Schlucklähmung. Gaumen unbeweglich. Zunge wird nicht mehr herausgestreckt. Incontinentia urinae et alvi. Nahrung kann nicht mehr genommen werden.

1. August. Befund wie gestern. Sprechen nicht mehr möglich. Athmung zuweilen aussetzend, schnarchend.

2. August. Heate Morgen unter zunehmender Athmungsinsufficienz (Cheyne-Stokes) Exitus.

Nach diesem Krankheitsverlauf handelte es sich um eine 48jährige Frau, welche offenbar an einerziemlich vorgeschrittenen Tabes (Stadium paralyticum) litt. Diese Frau ging binnen 8 Tagen (26. Juli bis 2. August 1903) an acut einsetzenden Lähmungserscheinungen zu Grunde, und zwar war die Lähmung aufsteigend, ergriff zunächst.die ohnehin schon paretischen Beine, griff über auf Rumpf, Arme und zuletzt auf die Hirnnerven. Eine Athmungslähmung führte den Tod herbei. Unsere Diagnose lautete demgemäss: acute aufsteigende Landry'sche Spinalparalyse.

Die Section ergab an positiven Befunden: Schlaffe Herzmuskulatur, kleiner verkreideterHerd in der rechten Lungenspitze. Geringe Milzschwellung. die Untersuchung des Centralnervensystems zeigte Folgendes:

Meningen zart, nicht verwachsen. Im Gehirn ausser mässiger Hyperämie nichts Abnormes, insbesondere keine Blutungen. Auf Querscbnitten durch die Medulla oblongata ist überall deutliche Hyperämie zu sehen. Stärkere Veränderungen kommen im Rückenmark vor:

Höhe des 1. Cervicalnerven: Vorderhörner verwischt, undeutlich. Deutliche Degeneration der Hinterstränge.

Halsanschwellung: Blutungen in der grauen Substanz, besonders in den Vorderhörnern. Degeneration der Hinterstränge.

1. Dorsalnerv: Blutungen in den Vorderhörnern. Diese erscheinen ansserdem grau verfärbt and verwaschen. Graue Flecke in den Hintersträngen.

6. Dorsalnerv: Grenze zwischen grauer und weisser Substanz undeutlich. Keine deutlichen Blutungen.

10. Dorsalnerv: Multiple kleinste Blutungen in der grauen Substanz. Degeneration der Hinterstränge.

Lendenanschwellung: Graue Substanz fleckig, verwaschen. Degeneration der Hinterstränge.

Unteres Lendenmark: Hyperämie und fleckige Degeneratiọn der Vorderhörner.

Cauda: ohne Abnormitäten.

Die hinteren und vorderen Wurzeln sind makroskopisch nicht deutlich verändert.

Die mikroskopische Untersuchung brachto zunächst die Bestätigung der schon makroskopisch festgestellten Degeneration der Hinterstränge und 
damit der klinischen Diagnose Tabes dorsalis: An Weigertpräparaten sieht man bereits vom unteren Lendenmark an schon makroskopisch und bei Lupenvergrösserung eine deutliche Abblassung der Goll'schen Stränge, die sich nach oben continuirlich fortsetzt, bis zum Nucleus fasciculi gracilis. Im Brustmark greift die Degeneration auf die peripheren $\Delta$ bschnitte der Burdach'schen Stränge über. Ausser dieser Frlkrankung der Hinterstränge findet sich vom unteren Brustmark an bis zur Medulla oblongata eine leichte Degeneration der Kleinhirnseitenstrangbahnen, mikroskopisch durch deutlichen Markscheidenschwund gekennzeichnet. In äbnlicher Weise sind auch die Gowers'schen Stränge beider Seiten vom unteren Brustmark an leicht erkrankt, schwerer afficirt (makroskopisch schon als helle Flecke zu sehen) erscheinen sie rom ersten Cervicalnerven ab bis hinein in die Medulla oblongata. Auch die spinale Trigeminuswurzel ist vom Beginn der Medulla ab degenerirt. Ferner sind im Brustmark. die Markscheiden der in den Clarke'schen Säulen verlaufenden markhaltigen Fasern gänzlich zu Grunde gegangen. Hier möchte ich auch erwähnen eine Sklerose mässigen Grades der Pyramidenseitenstränge im unteren Brustmark. Sehr ausgedehnt ist die Degeneration der hinteren eintretenden Wurzeln extra- und intramedullär, ferner der Lissauer'schen Randzone und der hinteren medialen Wurzelzone.

In allen den genannten Gebieten lässt sich durch van Giesonfärbung eine ziemlich beträchtliche Gliawucherung mit reichlichen Gliakernen und Corpora amylacea zur Anschaung bringen.

Neben diesen offenbar älteren Affectionen der sensiblen Bahnen und Wurzeln des Rückenmarks, wie sie bei vorgeschrittener Tabes häufig gefunden werden, bestanden nun noch zahlreiche anderweitige Veränderungen in Rückenmark und Medulla oblongata, die ich im Folgenden kurz schildern will.

Zur Anwendung kamen Färbungen mit $\mathrm{Hämalaun}$ allein und in Verbindung mit Eosin und van Gieson, ferner Weigert- und Marchifärbung.

March i schnitte aus Lendenanschwellung, Brustmark und Halsansch wellung zeigen übereinstimmend eine Erkranlkung besonders des grauen Querschnittantheils. Die graue Substanz ist dicht übersät mit schwarzen körnigen und fädigen Massen, schwarzen Schollen und Tropfen, zumal in der Gegend der Vorderund Seitenhörner und der vordeern Commissur. Ab und zu sieht man kleine tiefschwarze varicöse Fäßen das Gesichtsfeld durchzieben. Anbäufungen schwarzer Körner und Fäden sieht man, besonders in den Anschwellungen des Rückenmarks, im Verlaufe der aus den Vorderhörnern austretenden noch intramedullär gelegenen Wurzelfasern. Ebenso sind die extramedullären vorderen Wurzeln stark degenerirt. Hie und da ist im Bereich der Vorder- und Hinterbörner eine Körnchenzelle za sehen.

Weigertpräparate bestätigen diese Befunde allenthalben: die Markscheidenfärbung fehlt in den vorderen intra- und extramedullären Wurzein. Die graue Substanz ist fast völlig weiss geblieben. Diese Veränderungen sind bis zum Uebergang des Halsmarkes in die Medulla oblongata zu verfolgen.

Von sonstigen krankhaften Veränderungen in Rückenmark und Medulla obTongatasindzunennen: Blutungen und Erkrankung der Ganglienzellen. 
Die schon makroskopisch erkennbare Hyperämie wird durch die mikroskopische Untersuchung bestätigt: Sämmtliche Gefässe der Pia und der Marksubstanz sind prall gefüllt, die Wände der kleinsten Arterien sind häufig hyalin verändert. Es finden sich nun, wenn wir in aufsteigender Richtung vorgehen, sehr zahlyeiche Blutungen auf dem Querschnitt. Ueberall ist jedoch zu constatiren, dass die Blutungen in der Hauptsache im Gebiete der grauen Substanz gelegen sind, sowohl in Vorder- und Seiten- als auch Hinterhörnern. Wobl kommen auch einzelne kleinste Blutaustritte in der weissen Substanz vor; sie treten indessen gegenüber den zahlreichen und grossen Blutungen in der grauen Substanz ganz in den Hintergrund. Diese Blutangen siad am reichlichsten in der Cauda, im Lenden- und Halsmark. Sie fehlen völlig in der Medulla oblongata, ebenso im Brustmark in Hölıe des 6. Dorsalnerven, während sie im übrigen Brustmark nur spärlich sind. Im Lendenmark finden sich Blutungen in allen Theilen der grauen Substanz, am meisten aber in den Columnae laterales im Gebiete der grossen motorischen Ganglienzellen. Hier sind die Ganglienzellen häufig direct von rothen Blutkörperchen umgeben; diese füllen die pericellulären Räume zuweilen vollständig aus. Sonst liegren die rothen Blutkörperchen diffus über die graue Substanz verstreut oder in der Umgebung kleiner Arterien und Venen. Sie sind übrigens sämmtlich gut erhalten. Auch in der Umgebung des Centralcanals sind Blutungen häufig. Ferner finden sich Blutaustritte in der Fissura ant. med. und in den Scheiden der vorderen degenerirten Wurzeln.

Im Brustmark fehlen Blutungen in der weissen Substanz; in der grauen Substanz sind nur in Höhe des 1. Dorsalnerven in den Columnae lat. grössero Blutaustritte vorhanden, einige kleine in den Hinterhörnern. Im Halsmark kommen neben einigen kleinen subpial und in der weissen Substanz gelegenen Blutungen sehr zahlreiche in der grauen Substanz vor. Vornehmlich sind es hier die Columnae lat. und ant. mit ihren grossen Ganglienzellengruppen, welche die Blutungen enthalten. Blutungen in den Hinterhörnern sind in den verschiedenen Querschnittshöhen verschieden reichlich, fehlen z. B. in Höhe des 1. Cervicalmarks ganz. Am Uebergang des Halsmarks in die Medulla oblongata sind in den Columnae lat. nnd in den Hinterhörnern noch multiple Blutungen vorhanden, ebenso im Nucleus fasciculi gracilis. Auf Querschnitten durch die Medulla oblongata fehlen Blutungen.

Die unten näher zu beschreibenden Veränderungen der Ganglienzellen sind wiederum am ausgesprochensten im Lenden- und Halsmark, zumal im Gebiete der Anschwellungen. Unterhalb der Lendenanschwellung sind die Zellen der Vorderhörner gut erhalten, dje der Seitenhörner fast sämmtlich degenerirt, die Hinterhornzellen leidlich gut erhalten. In der Lendenanschwellung betrifft die Degeneration sämmtliche Ganglienzellen, relativ am wenigsten die Hinterhornzellen.

Wejt weniger ausgesprochen ist die Degeneration der Vorder- und Seitenhornzellen im unteren Brustmark; dagegen zeigen hier die Zellen der Clarkeschen Säulen tiefgehende Veränderungen. In Höhe des 6. Dorsalnerven sind 
nur geringfügige Veränderungen an den Zellen zu finden, stärkere schon wieder in Höhe des 1: Dorsalnerven.

In der Halsanschwellung sind die Zellen der. Vorder- und Hinterhörner etwa zur Hälfte, die Seitenhornzellen sämmtlich degenerirt. In Höhe des ersten Cervicalnerven sind die Ganglienzellen weit besser erhalten, als in der Halsanscbwellung, sind von normaler Grösse und Gestalt, mit rundem Kern und gut färbbaren, regelrecht angeordneten Nisslkörperchen. Auch am Uebergang des Rückenmarks in die Medulla oblongata sind die Zellen der Vorder- und Hinterhörner fast durchweg gut erhalten, nur wenige sind dentlich erlkrankt. Indessen sind hier die Zellen des Nucleus fasciculi gracilis erkranlit.

In der Medulla oblongata kommen wieder tiefgreifende Ganglienzeilenveränderungen vor, und zwar hauptsächlich in den Kernen des Nervus hypoglossus, ragus, glossopharyngeus und acusticus.

Die Degeneration der Ganglienzellen ist in allen Abschnitten des Markes durch die gleichen Veränderungen charakterisirt: Die erkrankten Zellen sind meist stark gequollen, um das 2-3fache vergrössert. Ibre Form ist rund, bauchig, oval, viereckig; ihre Fortsätze sind theils verloren gegangen, theils sind die abgehenden Achsencylinder gequollen, verdickt, varicös, vielfach gewunden. Während sich in den gut erhaltenen Zellen das T'igroid sehr gut färbt, ist das Tigroid der erkrankten Zellen auf dem gleichen Querschnitt in kleinste Körnchen aufgelöst, theils diffus über die ganze Zelle verstreui, theils der Peripherie des Zellleibs, theils dem Kern dicht angelagert, oft innig vermengt, mit den zuweilen diffus über die Zelle verstreuten Pigmentkörnchen. In vielen Zellen ist gar kein Tigroid zu sehen. Der Kern fehlt häufig. Wo er erhalten ist, ist er an die Peripherie gerückt, deformirt, geschrumpft, eckig. Das Kernkörperchen ist meist erhalten. Hie und da sind im Protoplasma eine oder mehrere Vacuolen zu sehen. Von einigen Zellen sind nur noch grosse hyaline unregelmässig gestaltete, structurlose Schollen übrig geblieben.

SchIiesslich möchte ich noch als unwesentliche Nebenbefunde einige Abnormitäten erwähnen: Der Centralcanal, dessen Epithel überall gut erhalten jst, zeigt an vielen Stellen dünne seitliche Ausläufer, die nach oben oder unten zu ausbiegen. Auf solchen Querschnitten wird dadurch ein doppelter Centralcanal vorgetäuscht. Sodann findet sich im Lendenmark im medialen Theil des einen Hinterstrangs ein kleiner versprengter Herd grauer Substanz mit einer Blutung. Im mittleren Brustmark kommen im Hinterstrang einer Seite versprengte Ganglienzellen vor.

Wenn wir nochmals kurz recapituliren, so handelte es sich um eine an Tabes dorsalis leidende Frau, welche innerhalb von 8 Tagen unter den Erscheinungen der Landry'schen Paralyse zu Grunde ging.

Darüber, ob dieser Fall klinisch mit Recht der Landry'schen Paralyse zugezählt wird, sind Zweifel wohl nicht möglich. Typisch ist der fieberlose, rasch aufsteigende Verlauf der Lähmung, abschliessend mit Athmungslähmung. Die Lähmung war klinisch eine rein motorische, 
schlaffe. Gegen Ende traten Blasen- und Mastdarmstörungen auf. Muskelatrophien waren bei dem schnellen Verlauf erklärlicher Weise nicht zu constatiren. Das Sensorium blieb fast bis zuletzt frei. Die Sensibilitätsstörungen sowie das Fehlen der Sehnenreflexe waren auf Rechnung der Tabes zu setzen. Eine elektrische Untersuchung der Muskeln war leider unterblieben. Dieser Verlauf stimmt durchaus mit den von den verschiedenen Autoren gegebenen Schilderungen [z. B. bei Oppenheim $\left.\left.{ }^{1}\right)\right]$ überein.

Interessant und meines Wissens nach nicht beobachtet war die Complication mit Tabes dorsalis.

Dass die Diagnose Tabes durch die makroskopische und mikroskopische Untersuchung Bestätigung findet, habe ich bereits erwähnt. Die in den betreffenden Degenerationsgebieten vorhandene Gliawucherung zeigt, dass die Veränderungen älteren Datums sind. Auch die in den Pyramidenseitensträngen auf eine kurze Strecke gefundene Degeneration erweist sich wegen der Gliavermehrung als älterer Process; viellëicht ist hierauf die bei der Aufnahme bereits bestehende Parese der Beine zn beziehen.

Das anatomische Substrat für die aufsteigende Lühmung ist eine acute Erkrankung der grauen Substanz in allen Theilen des Rückenmarks und der Medulla oblongata, gekennzeichnet durch Blutungen, Schwund der Markscheiden, Degeneration der Ganglienzellen mit consecutiver Degeneration der vorderen Wurzeln. Am stärksten sind durchweg die Vorder- und Seitenhörner mit den von ibnen ausstrahlenden motorischen Wurzelfasern, besonders in Hals- und Lendenmark, ergriffen, aber auch die Hinterhörner sind wesentlich betheiligt, vornehmlich in Bezug auf ihre Ganglienzellen (Clarke'sche Säulen). In der Medulla oblongata kennzeichnet sich die Erkrankung durch Degeneration der Ganglienzellen in den grauen Kernen der genannten Nerven.

Es handelt sich also um eine Poliomyelitis acuta diffusa resp. disseminata, wobei der motorische Antheil der grauen Substanz stärker betheiligt ist als der sensible. Diese Befunde erklären den klinischen schnellen Verlauf zur Genüge. Die Erkrankung hat offenbar im Lendenmark begonnen, ist schnell aufgestiegen und hat zuletzt die Medulla oblongata befallen; wenigstens zeigt diese die geringsten Veränderungen, insbesondere kleine Blutungen.

Diese Untersuchungsergebnisse sind beachtenswerth im Hinblick besonders auf das in neuerer Zeit auftretende Bestreben, die Landry'sche Paralyse unter die Polyneuritis acuta einzureihen (Rolly). Leider

1) 1. c. 
musste in unserem Falle aus äusseren Gründen eine Untersuchung der peripheren Nerven unterbleiben, was ja zweifellos als ein Mangel empfunden werden muss. Indessen glaube ich nicht, dass eine diesbezügliche Untersuchung in unserem Falle Wesentliches zu Tage gefördert haben würde. Einmal waren klinisch keinerlei Symptome einer Polyneuritis wie bei den Fällen Rolly's ${ }^{1}$ ) vorhanden. Sodann aber würde man Befunden von Degenerationen in den pheripheren Nerven keine allzu grosse Bedeutung beimessen können, da bekanntlich auch bei Tabes dorsalis sehr häufig periphere neuritische Veränderungen gefunden worden sind. Es wäre jedenfalls die Entscheidung, ob man diese auf die Tabes oder auf die Landry'sche Paralyse zu beziehen gehabt hätte, in unserem Falle schwierig und nicht einwandsfrei gewesen. Da sich sämmtliche klinische Erscheinungen zwanglos aus unseren Befunden erklären lassen, so wird die Annahme, dass in unserem Falle eine Neuritis vorausgegangen sein müsste und dass der Process auf dem Wege der peripheren Nerven anf das Rückenmark übergegriffen habe, eine Möglichkeit, die z. B. von Leyden ${ }^{2}$ ) und $\mathrm{Krewer}{ }^{3}$ ) betont wird, durch nichts gestützt. Sie wird auch durch die Untersuchungen Arneth's 4 ) unwahrscheinlich.

So würde denn unsere Beobachtung wiederum die Zabl der Fälle vermehren, bei denen in Rückenmark und Medulla oblongata palpable Läsionen myelitischer Natur gefunden wurden.

Unter den zahlreichen Fällen dieser Art stimmen unsere Befunde am meisten mit denen von $\operatorname{Immermann^{5}}$ ) und $\mathrm{Hlawa}{ }^{6}$ ) überein. Immermann fand eine Poliomyelitis ant. acuta: makroskopisch Degenerationen in den grauen Vorderhörnern, im Lendenmark in Gestalt ziegelrother Flecken, die im Brustmark mehr verwaschen waren. Im unteren Brustmark fanden sich auch in den centralen Regionen der grauen Substanz Degenerationen: Im oberen Brust- und unteren Halsmark war die Degeneration wieder nur auf die Vorderhörner beschränkt, im oberen Halsmark war der Querschnitt normal; mikroskopisch starke Gefässinjectionen, reichliche Anhäufung von Körnchenzellen, besonders längs der Gefässe, Ganglienzellen zum Theil erhaiten, zum Theil untergegangen und durch hyaline Massen ersetzt. Nach Immermann beweist
1) 1. c.
2) 1. c. S. 26
3) Zeitschrift f. klin. Med. Bd. 32.
4) $1 . \mathrm{ce}$.
5) Archiv für Psych. Bd. 16. 1885. S. 848.
6) Cit. nach Leyden 1. c. S. 24. 
434 Dr. Hans Lohrisch, Ein Beitr. z. path. Anat. d. Landry'schen Paralyse.

der Fall, dass es eine Form der Poliomyelitis ant. acuta giebt, die dem Bilde der Landry'schen Paralyse entspricht, und dass Landry'sche Paralyse und Poliomyelitis ant. acuta nur verschiedene Grade einer nosologischen Einheit darstellen.

Noch mehr Aebnlichkeiten bietet der Fall von $\mathrm{Hlaw}$ a. Hier fand sich eine Poliomyelitis acuta disseminata, partialiter haemorrhagica; makroskopisch Hyperämie der gesammten grauen Substanz, ferner iom Lendentbeil bis zum Bulbus acute, zum Theil hämorrhagische Entzündung in Vorder- und Hinterbörnern, selbst in den Clarke'schen Säulen; mikroskopisch wurde dieser Befund bestätigt, die Ganglienzellen waren stellenweise nicht zu erkennen. Die peripheren Nerven wurden intact gefunden.

Das bisher gesammelte Material erlaubt meines Erachtens immer noch nicht, klinisch sichere Falle Landry'scher Paralyse alusschliesslich unter die Myelitis oder, wie Rolly ${ }^{1}$ ) will, aussehliesslich unter die Polyneuritis acuta einzureihen. Dazu sind die Beobachtungen zu mannigfach und einander widersprechend. Am plausibelsten erscheint vorläufig der oben citirte Standpunkt Soltmann's ${ }^{2}$ ) und Oppenheim's ${ }^{3}$ ). Nur hinsichtlich der Aetiologie herrscht bei sämmtlichen Autoren Uebereinstimmung, insofern sie die Landry'sche Paralyse mit Sicherheit als eine auf Infection resp. Intoxication beruhende Erkrankung auffassen. Bezüglich der Art dieser Infection oder Intoxication ist unser Fall allerdings ebenso wenig klargestellt wie die meisten anderen Fälle. Bacterienbefunde konnte ich im Rückenmark nicht erheben. $O b$ Lues und Alcohol, welche beiden Momente bei unserer Patientin nicht auszuschliessen waren, in der Aetiologie der Landry'scben Paralyse eine Rolle spielen, ist noch unbewiesen.

Zum Schlusse spreche ich Herrn Prof. Dr. Schmidt für die Ueberlassung des Falles meinen verbindlichsten Dank aus.
1) 1. c.
2) 1. c.
3) l. c. 